\title{
Stimulus control by dot position in pigeons
}

\author{
G. WILLIAM FARTHING \\ University of Maine, Orono, Maine 00473
}

\begin{abstract}
In Experiment I positive dot-position generalization gradients were obtained following training in which pecks at a white key with a black dot in a fixed position (at either the top, bottom, left, right, or center of the key) were sometimes reinforced $\left(S^{+}\right)$and pecks at a blank white key were never reinforced (S-). Single-stimulus training was not sufficient to establish control by dot position. In Experiment II negative dot-position gradients were obtained following training on a discrimination between $\mathrm{S}+$ blank vs. $\mathrm{S}$ - dot. Implications of these and other results for excitatory and inhibitory stimulus control were discussed.
\end{abstract}

The main purpose of this study was to determine whether the pecking response of pigeons will come under the control of the spatial position of a black dot on the response key. The question is of interest in its own right, as part of the more general question of which stimulus dimensions pigeons attend to without special discrimination training, and which dimensions acquire control only after discrimination training. For example, nondifferential training is sufficient to establish control of the pigeon's pecking response by the wavelength (e.g., Guttman \& Kalish, 1956) and line tilt (e.g., Hearst, Koresko, \& Poppen, 1964) dimensions. On the other hand, control by the orientation of a parallelogram (Vetter \& Hearst, 1968), tone frequency (Jenkins \& Harrison, 1960), or click frequency (Farthing \& Hearst, 1972) dimensions is typically obtained only following explicit discrimination training.

A study of stimulus control by dot position might be particularly interesting since it is conceivable that pigeons would have a strong tendency to peck at small black dots on a lighted response key, regardless of their specific location on the key. Part of the folklore of many operant conditioning laboratories is that it is easier to shape the keypecking response in pigeons if a dot is drawn on the key with a pencil than if a plain white or colored key is used (Ferster \& Skinner, 1957, p. 31). Gardner (1970) has shown that a dot on the key facilitates autoshaping in quail. Presumably the dot facilitates shaping because it resembles a piece of grain. If pigeons have a strong tendency to peck at small, black dots, then they might be relatively inattentive to the position of the dot on the key.

A second reason for studying stimulus control by dot position is its possible relevance to the problem of the nature of the controlling stimulus dimension in experiments involving changes in the orientation, shape, or size of a geometric figure. Apparent stimulus control by one of these dimensions of a geometric figure might

This research was supported by NIMH Grant No. 18290. The author thanks Tom Nelson and Mike Opuda for assistance in various phases of the project. Reprints may be obtained from the author at the Department of Psychology, 301 Little Hall, University of Maine, Orono, Maine 04473. actually be due to control by the spatial position of a small part of the figure, such as a corner or edge. A demonstration of stimulus control by the position of an isolated dot would make such an interpretation plausible and would suggest the value of further analyzing the effective controlling stimulus dimension in studies where the orientation, shape, or size of a geometric figure are varied.

\section{EXPERIMENT I}

\section{Subjects}

Ten Silver King pigeons (about 6 years old) were maintained at $75 \% \pm 15 \mathrm{~g}$ of their free-feeding body weights. The birds had previously been used in an auditory discrimination experiment in which pecks at a red key were sometimes reinforced on a variable-interval schedule.

\section{Apparatus}

The birds were trained in a Lehigh Valley Electronics pigeon test chamber (Model 1519), in which the transparent plastic response key was mounted behind a 25 -mm-diam opening which was centered above the square feeder opening, and about $25 \mathrm{~cm}$ above the floor. An in-line digital display cell (Industrial Electronics Engineers special pattern; manufacturer's identification No. 84858, with No. 44 miniature bulbs) could transilluminate the key with stimuli which consisted of either a plain white field (blank), or a white field with a black dot on it. The dot was $5 \mathrm{~mm}$ in dia, and was positioned either at the center of the key or at $5 \mathrm{~mm}$ (center to edge) from either the top, bottom, right, or left edge of the key. A houselight was mounted about $6 \mathrm{~cm}$ above the response key. External noises were masked by an air blower in the test chamber and a white noise in the test chamber room.

\section{Procedure}

Design. Following initial single-stimulus (nondifferential) training, one group of birds was trained with S+ dot vs. S- blank (no dot), while matched subjects received additional single-stimulus training with $\mathrm{S}+$ only. A different dot position (top, bottom, left, right, center) served as $\mathrm{S}+$ for each pair of subjects.

Training. Each bird was initially placed in the test chamber with the houselight on and the S+ on the key. In some cases it was necessary to reshape the peck response. Once the bird started pecking the key, every peck was reinforced until 30 reinforcers were collected. Reinforcers consisted of 3-sec access to mixed grain. During this period of continuous reinforcement 


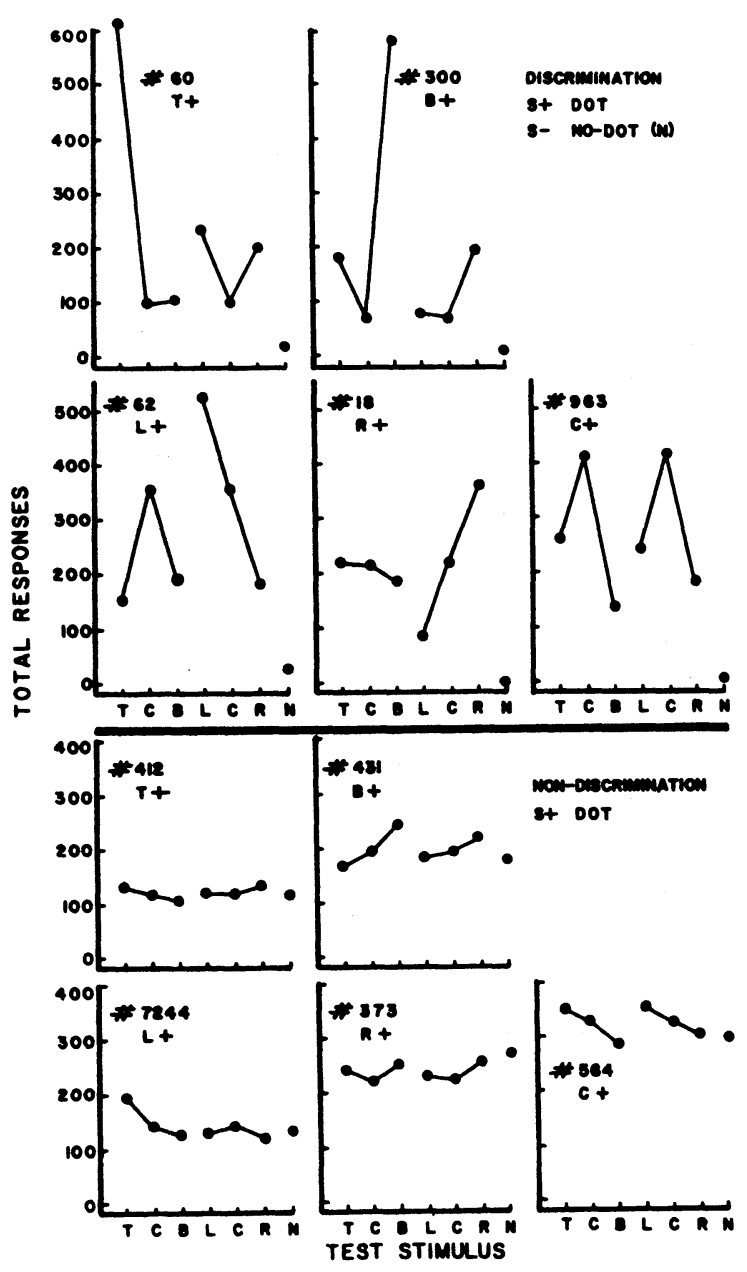

Figure 1. Generalization test data for Experiment l. Birds in the top panel were trained on a discrimination between $S+\operatorname{dot}$ vs. S- blank (no dot), whereas those in the bottom panel received only single-stimulus training with $S+$ dot. The test stimuli included dots in the top (T), bottom (B), left $(L)$, right (R), and center (C) positions on the key, and a blank stimulus with no $\operatorname{dot}(\mathrm{N})$. The position of the $\mathrm{S}+\operatorname{dot}$ is indicated for each subject.

(CRF), the houselight was faded out, and it remained off for the duration of the experiment.

All birds were then given seven sessions of single-stimulus training, during which pecks at $\mathrm{S}+$ were reinforced on a one-minute variable-interval schedule (VI 1-min). Each single-stimulus training session consisted of 6030 -sec stimulus presentations. Stimulus presentations were separated by $10-\mathrm{sec}$ blackouts, during which the stimulus was off and responses were not reinforced.

Following single-stimulus training, one bird of each pair was trained on a discrimination involving $\mathrm{S}+$ dot (VI 1-min) vs. Sblank (extinction). The discrimination birds were trained until they met a criterion of two successive sessions in which the response rate in S-was not more than $10 \%$ of the rate in S+. The matched nondiscrimination birds were given additional single-stimulus training for the same number of sessions that the discrimination partner was run. In these additional single-stimulus training sessions, the number and temporal patterning of $\mathrm{S}+$ presentations was the same as for the discrimination birds, but S- presentations were replaced by 30-sec extended blackouts. Discrimination sessions consisted of a pseudorandom mixture of $30 \mathrm{~S}+$ and $30 \mathrm{~S}$-presentations, with not more than three of one type of stimulus in succession. Stimulus presentations were separated by 10 -sec blackouts.

Testing. Following the last training sessions, each bird was given a warmup period equivalent to one-third of a trainin! session, followed by a generalization test in extinction. The six test stimuli were the blank and the single dots presented in top, bottom, left, right, and center positions. The test stimuli were presented once in each of 12 randomized blocks. Five different test stimulus sequences were used, with each pair of birds getting a different sequence. Test stimulus presentations were of 30 -sec duration and were separated by $10-\mathrm{sec}$ blackouts. The experimenter recorded the number of pecks during each test stimulus presentation.

\section{Results}

Figure 1 shows the number of pecks at each test stimulus, for each individual subject. The discrimination group is in the top panel, and the nondiscrimination group is in the bottom panel. In order to facilitate comparison of the response totals to the three dots in the vertical row (top, center, bottom) and the horizontal row (left, center, right), the total for the center dot is plotted twice.

None of the birds given only single-stimulus training had a clear-cut maximum response rate at their training stimulus (though Bird 431 pecked somewhat more with the dot at the training position than with it at other positions). ${ }^{1}$ On the other hand, all of the birds with discrimination training pecked much more at their S+ dot than at other dot position stimuli. Also, all of the discrimination birds pecked at the blank $(\mathrm{N})$ stimulus much less than at any of the dot stimuli, though this was not true of the birds given only nondifferential training.

Jenkins and Sainsbury (1970) have shown that during discrimination training in which a black dot appeared only on S+ trials, pigeons tended to peck directly at the dot. Informal observations during the present experiment revealed that birds in the discrimination group usually pecked directly at the dot during discrimination training. The orientation of the birds' pecks in relation to the dot was not observed during the generalization test.

\section{Discussion}

In Experiment I the pecking behavior of pigeons was not controlled by the position of the dot following single-stimulus training with a black dot in a fixed position on a white key. In fact, the single-stimulus-trained subjects pecked about as much at the blank (no dot) stimulus as they did at the dot stimuli. However, pecking was controlled by dot position following discrimination training between a dot in a fixed position $(\mathrm{S}+)$ and blank (S-). Explicit discrimination training between dots in two different positions was not necessary in order to establish control by dot position.

It could be argued that the failure of dot position to control the pecking response in the nondifferentially-trained birds was due to the prior training of these subjects in an experiment which involved reinforcement of pecks at a plain red key. This possibility cannot be ruled out on the basis of the present data. However, acquisition of control by line tilt, during 
single-stimulus training with a vertical black line on a white background, is not prevented by prior training with either a plain white key (Honig, 1970, Figure 9.6) or a plain blue key (Farthing, unpublished data). It seems unlikely that prior training with a red key was responsible for the absence of control by dot position in the nondifferentially-trained birds in Experiment I.

\section{EXPERIMENT II}

Several experiments have shown negative line tilt generalization gradients, with a minimum at S-, following discrimination training with a black vertical line on a white background as S- and a blank white key (no line) as S+ (e.g., Farthing \& Hearst, 1968). Experiment II was an attempt to determine whether pigeons would show negative dot position generalization gradients, following training with a dot in one position on a white key as S- vs. a blank white key (no dot) as S+. This experiment included a control group given nondifferential training with S+ blank only, prior to the dot position generalization test. The purpose of this control group was (a) to determine whether pigeons tend to peck any more, or less, at dots in one position on the key than at dots in other positions, where they have not had any prior exposure to dots on the key, and (b) to provide a baseline against which to evaluate possible suppressive inhibition of responding by the $S$ - dot in the discrimination group (Hearst, Besley, \& Farthing, 1970).

\section{Method}

Subjects and apparatus. Ten Silver King pigeons were maintained at $75 \% \pm 15 \mathrm{~g}$ of their free-feeding body weights. Five of the birds had previously been used in an auditory discrimination experiment in which pecks at a red response key were sometimes reinforced on a variable-interval schedule. The other five birds (indicated by asterisks in Figure 2) were experimentally naive. The apparatus was the same as in Experiment I.

Procedure. Following initial single-stimulus training with a plain white key, five of the birds were trained on a discrimination between S+ blank (no dot) vs. S- dot. The dot was in a different position (top, bottom, left, right, center) for each discrimination-trained bird. Control birds received approximately the same number of training sessions as matched discrimination subjects, but in place of the S-presentations the control birds had extended 30 -sec blackouts. Other details of training and testing were the same as in Experiment $I$.

\section{Results}

The discrimination-trained birds met the discrimination criterion in a mean of 13.2 sessions, which was not significantly different from the 11.0 sessions required by the dot-positive discrimination-trained birds in Experiment $I$.

Dot-position generalization test data are shown in Figure 2. All of the discrimination-trained birds showed stimulus control by dot position. The minimum of the dot-position gradient was at the S- dot for three birds (birds 032, 123, and 275) while for the other two birds the minimum was at an adjacent dot and $\mathrm{S}$ - received the second-lowest number of pecks. All four of the birds with an S- dot at an edge position (top, bottom, left, or right) pecked more with the dot at the edge position opposite the S-position than with the dot in any other position.

The control birds, trained with S+ blank only, showed little or no control by dot position. ${ }^{2}$ There was no systematic tendency of the control birds to peck at a dot in one particular position more, or less, than dots in other positions. On the other hand, there is evidence that the control birds did notice the dots, since all of them pecked more at $\mathrm{S}+$ blank $(\mathrm{N})$ than at any of the dot stimuli.

In the discrimination group, the number of pecks at the S- dot ranged from 3 to 42 (mean 16.8), whereas in the control group the number of pecks at the dot stimulus with the fewest pecks ranged from 77 to 234 (mean 122.8); this difference is statistically significant ( $\mathrm{U}=0, \mathrm{p}<.01$ by a two-tailed test).

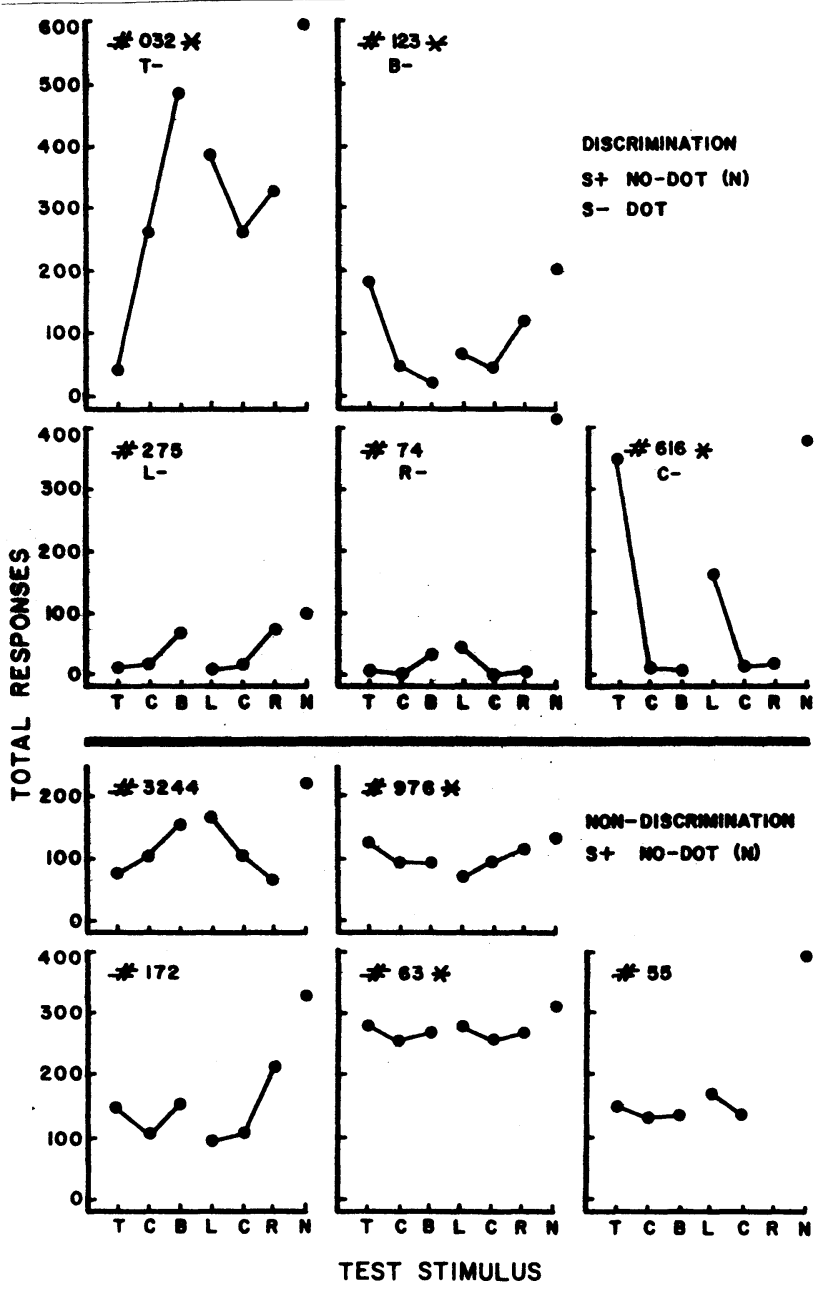

Figure 2. Generalization test data for Experiment II. Birds in the top panel were trained on a discrimination between $S+$ blank (no dot) vs. S- dot, whereas those in the bottom panel received only single-stimulus training with $S+$ blank. The position of the S- dot is indicated for each subject. ${ }^{2}$ 


\section{Discussion}

Experiment II demonstrates negative generalization gradients for dot position. Following discrimination training between $\mathrm{S}+$ blank vs. S- dot, the minimum of the generalization gradient was at the S- dot position for most of the birds, and for the other birds the $\mathrm{S}$ - position received the second-lowest number of pecks. The control group, trained with $\mathrm{S}+$ blank only, demonstrated that there is no consistent tendency for birds withour prior exposure to dots on the key to peck any more, or less, at a dot in any one particular position than at dots in other positions. (It is interesting that addition of dot to blank in the Experiment II control group resulted in a generalization decrement, while removal of the dot in the Experiment I control group did not produce a consistent generalization decrement. Speculation as to the cause of this asymmetry will be withheld. However, these data suggest that, if pigeons do have any innate tendency to pick at small dots which resemble grain, then the dots used in this study were apparently inadequate to engender such pecking.)

Negative generalization gradients on a dimension of S- have previously been accepted as evidence for inhibitory stimulus control (Jenkins, 1965; Hearst et al., 1970) when such gradients have been obtained following discrimination training in which S+ is orthogonal to the dimension of the generalization test. One might argue that blank (no dot) is orthogonal to the dot position continuum. That is, for example, a plain white key with no dot is no more similar to a white key with a dot at the top than it is to a white key with a dot at the bottom. Although blank may be logically orthogonal to dot position, this orthogonality may be destroyed by discrimination training in which the dot always occurs at the same position. If the dot always appears in the same position, when it appears, then the bird might learn to look predominantly at that particular position on the key. Thus, for example, in a discrimination between S+blank vs. S- top dot, the effective discrimination learned by the subject might be: $\mathrm{S}+$ (white space at top of key) vs. S- (dark spot at top of key). If this were the case, then during the generalization test, when the dot is moved away from the top position the stimulus appears to the subject to be more similar to $\mathrm{S}+$, as well as less similar to $\mathrm{S}-$, because moving the dot away from the top of the key leaves white space at the top of the key (like the effective $\mathrm{S}+$ ). If this argument is correct, then the negative dot position gradients in Experiment II are not pure gradients of inhibition, but probably reflect a combination of excitatory and inhibitory stimulus control. Both types of control were probably involved also in the positive dot position gradients demonstrated in Experiment $I$.

Hearst et al. (1970, p. 36) have made a similar argument regarding the possible controlling stimulus dimension in studies of gradients of inhibition on a line tilt continuum, following training with $\mathrm{S}+$ blank vs. S- vertical line. Perhaps during such training the birds learn to look predominantly at an area near the top (or bottom) of the key, and thus learn an effective discrimination based on the presence or absence of blank space vs. a dark spot at the top (or bottom) of the key. In the generalization test, as the line is tilted away from vertical the top (or bottom) of the key comes to look more like S+, as well as less like S-.

In order to test the plausibility of this explanation of line tilt control, the author conducted an additional study in which pigeons learned a discrimination between $\mathrm{S}+$ (a row of three dots across the key) vs. S- (blank). The basic procedure was identical to that for the discrimination groups in Experiments I and II The $S+$ was a vertical dot row (dots in the top, center, and bottoin positions) for eight birds, while for another eight birds S+ was a horizontal dot row (left, center, and right positions). Following discrimination acquisition, all of the birds were given a generalization test which included single dots in the five different positions. It was predicted that, if the dot row discrimination was learned on the basis of the presence or absence of a dot in a position corresponding to one end of the $\mathrm{S}+$ dot row, then the birds would respond more to a test stimulus with a single dot in one of the $\mathrm{S}+$ rowend positions than to single-dot stimuli in other positions. As it turned out, 11 of the 16 birds pecked more with a single dot in the center position than with the dot in other positions $(8$ of these 11 birds had their second-greatest response total with the dot in a S+ rowend position). The remaining 5 birds all pecked more with the single dot in a $\mathrm{S}+$ row end position, thus conforming to the prediction (4 of these 5 birds had their second-greatest response total with the dot in the center position). These data suggest that pigeons learning a presence vs. absence discrimination involving a vertical or horizontal dot row attend primarily to the center of the dot row, or at least to the center position plus an end position, rather than merely to a position near one edge of the key. To the extent that the results of this experiment with dot rows are generalizable to experiments with line tilts, they suggest that it is safe for experimenters to continue to assume a functional orthogonality between line presence vs. line absence in studies of excitatory and inhibitory line tilt control.

\section{NOTES}

1. It might be more than a coincidence that the only single-stimulus-trained bird with a generalization gradient peak at its training stimulus was the bird trained with the dot in the bottom position; we have observed that most pigeons tend to peck mostly at the bottom portion of the key in our test chambers.

2. Near the beginning of Bird 55's generalization test the projector bulb for the right dot stimulus (R) was accidentally burned out. Consequently this stimulus was omitted from the test.

\section{REFERENCES}

Farthing, G. W., \& Hearst, E. Generalization gradients of inhibition atter different amounts of training. Journal of the Experimental Analysis of Behavior, 1968, 11, 743-752.

Farthing, G. W., \& Hearst, E. Stimulus generalization and discrimination along the click-frequency (flutter) continuum in pigeons. Perception \& Psychophysics, 1972, 12, 176-182.

Ferster, C. B., \& Skinner, B. F. Schedules of reinforcement. New York: Appleton-Century-Crofts, 1957.

Gardner, W. M. An analysis of auto-shaping. Paper presented at the convention of the Southeastern Psychological Association, 1970.

Guttman, N., \& Kalish, H. I. Discriminability and stimulus generalization. Journal of Experimental Psychology, 1956, 51, 79-88.

Hearst, E., Koresko, M. B., \& Poppen, R. Stimulus generalization and the response-reinforcer contingency. Journal of the Experimental Analysis of Behavior, 1964, 7, 369-380.

Hearst, E., Besley, S. \& Farthing, G. W. Inhibition and the stimulus control of operant behavior. Journal of the Experimental Analy sis of Behavior, 1970, 14, 373-409.

Honig, W. K. Attention and the modification of stimulus control. In D. I. Mostofsky (Ed.), Attention: Contemporary theory and analysis. New York: Appleton-Century-Crofts, 1970. Pp. 193-238.

Jenkins, H. M. Generalization gradients and the concept of inhibition. In D. Mostofsky (Ed.), Stimulus generalization. Palo Alto: Stanford University Press, 1965. Pp. 55-61.

Jenkins, H. M., \& Harrison, R. H. Effect of discrimination training on auditory generalization. Journal of Experimental Psy chology, 1960, 59, 246-253.

Jenkins, H. M., \& Sainsbury, R. S. Discrimination learning with the distinctive feature on positive or negative trials. In $D$. I Mostofsky (Ed.) Attention: Contemporary theory and

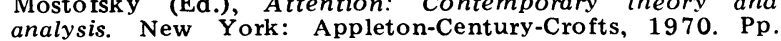
239-273.

Vetter, G., \& Hearst, E. Generalization and discrimination of shape orientation in the pigeon. Journal of the Experimental Analysis of Behavior, 1968, 11, 753-765.

(Received for publication May 19, 1975.) 